\title{
On the Output Controllability of Positive Discrete Linear Delay Systems
}

\author{
Mouhcine Naim, ${ }^{1}$ Fouad Lahmidi, ${ }^{1}$ Abdelwahed Namir, ${ }^{2}$ and Mostafa Rachik ${ }^{1}$ \\ ${ }^{1}$ Laboratory of Analysis, Modeling and Simulation, Faculty of Sciences Ben M'sik, Hassan II University, Casablanca, Morocco \\ ${ }^{2}$ Laboratory of Information Technology and Modeling, Faculty of Sciences Ben M'sik, Hassan II University, Casablanca, Morocco
}

Correspondence should be addressed to Mouhcine Naim; naimmouhcine2013@gmail.com

Received 15 August 2017; Revised 30 September 2017; Accepted 9 October 2017; Published 23 November 2017

Academic Editor: Valery Y. Glizer

Copyright (c) 2017 Mouhcine Naim et al. This is an open access article distributed under the Creative Commons Attribution License, which permits unrestricted use, distribution, and reproduction in any medium, provided the original work is properly cited.

Necessary and sufficient conditions for output reachability and null output controllability of positive linear discrete systems with delays in state, input, and output are established. It is also shown that output reachability and null output controllability together imply output controllability.

\section{Introduction}

The research devoted to controllability was started by Kalman in the 1960s [1] and refers to linear dynamical systems. Controllability is one of the fundamental concepts in the modern mathematical control theory $([2-4], \ldots)$ and continually appears as a necessary condition for the existence of solutions to many control problems, for example, stabilization of unstable system by feedback and optimal control. Basically a system is controllable if it is possible to transfer it around its entire configuration space using only certain admissible controls. There exist many definitions of controllability that depends on the framework or the class of models applied. The following are examples of variations of controllability notions which have been introduced in the control literature: asymptotic controllability [5], relative controllability [6], constrained controllability [7], complete controllability [8], approximate controllability [9], small controllability [10], output controllability $[11,12]$, and so on.

In most engineering applications, it is needed to direct the output toward some desired value. In fact, having control over the output of the system has a significant importance if not more than the states. For example, the control of a multilink cable-driven manipulator, where the task is typically defined in terms of end effector pose, rather than the joint positions and velocities which can define the system's state [13], also, controlling the output of fixed-speed wind turbines in the electrical network, which can directly affect the behavior of power systems [14]. Output controllability is a property of the impulse response matrix of a linear invariant-time system which reflects the dominant ability of an external input to move the output from any initial condition to any final condition in a finite time [2]. In general, the output controllability means that the system's output can be directed regardless of its state [15]. The necessary and sufficient criterion for output controllability of linear time-invariant systems is addressed in, for example, [12].

Positive systems are a wide class of systems in which state variables and outputs are constrained to be positive, or at least nonnegative for all time whenever the initial conditions and inputs are nonnegative. Since the state variables and outputs of many real-world processes represent quantities that may not have meaning unless they are nonnegative because they measure concentrations, numbers, populations, and so on, positive systems arise frequently in mathematical modeling of engineering problems, management sciences, economics, social sciences, chemistry, biology, ecology, pharmacology, medicine, and so forth.

An excellent survey of positive systems with an emphasis on their applications in the areas of management and social sciences is given by Luenberger in [16]. The more recent monographs by Farina and Rinaldi in [17] and Kaczorek in [18] are devoted entirely to positive linear systems and some of their applications. Since positive systems are confined 
within a cone located in the positive orthant rather than in the whole space $[19,20]$, their analysis and synthesis are more complicated and more challenging.

The state controllability of positive linear discrete systems is largely studied by several authors since late 1980s [21-26], the problem of controllability of linear positive discrete systems with delays in state or control was discussed in [27]. The problem of output reachability of positive linear discrete systems is addressed in [28]. The output reachability of positive discrete linear systems with state delay has been studied in [29].

In this paper we examine the issue of output reachability, null output controllability, and output controllability for positive linear systems with multiple delays in state, input, and output. These concepts are equivalent for unconstrained systems. The output reachability of discrete positive linear systems are characterized and proven by a simple algebraic proof. The criteria for the null output controllability will be established. We show that these properties are not equivalent for positive systems. In addition we prove that the positive system is output controllable only if it is output reachable and null output controllable.

The structure of the paper is as follows. In the next section some mathematical preliminaries of positive linear discrete systems with delays are presented. We investigate the output reachability and null output controllability of positive linear discrete systems with delays in state, input, and output, respectively, in Sections 3 and 4. In Section 5, necessary and sufficient conditions for the output controllability of positive delay systems are provided. Numerical examples will be presented in Section 6.

\section{Preliminaries}

First we introduce some notations. $\mathbb{N}$ is the set of nonnegative integers, $\mathbb{N}_{+}$the set of positive integers, $\sigma_{s}^{k}=\{s, s+1, \ldots, k\}$ the finite subset of $\mathbb{N}$ with $s \leq k, \mathbb{R}^{n}$ the set of real vectors with $n$ components, and $\mathbb{R}_{+}^{n}$ the set of vectors in $\mathbb{R}^{n}$ with nonnegative components; that is,

$$
\mathbb{R}_{+}^{n}=\left\{x=\left(x_{1}, x_{2}, \ldots, x_{n}\right)^{T} \in \mathbb{R}^{n}: x_{i} \geq 0, i \in \sigma_{1}^{n}\right\},
$$

where $T$ denotes the transpose, $\mathbb{R}^{n \times m}$ the set of real matrices of order $n \times m\left(\mathbb{R}^{n}=\mathbb{R}^{n \times 1}\right), I_{n}$ the identity matrix in $\mathbb{R}^{n \times n}$, and $A^{-1}$ the inverse of $A \in \mathbb{R}^{n \times n}$.

In this work, we consider the discrete linear delay system

$$
\begin{aligned}
& x_{i+1}=\sum_{j=0}^{p} A_{j} x_{i-j}+\sum_{j=0}^{q} B_{j} u_{i-j}, \quad i \in \mathbb{N}, \\
& u_{-j} \in \mathbb{R}^{m} \text { for } j \in \sigma_{1}^{q}, \\
& x_{-j} \in \mathbb{R}^{n} \text { for } j \in \sigma_{0}^{p},
\end{aligned}
$$

with the output equation

$$
y_{i}=\sum_{j=0}^{l} C_{j} x_{i-j}+\sum_{j=0}^{v} D_{j} u_{i-j}, \quad i \in \mathbb{N}, \text { with } l \leq p, v \leq q
$$

where $x_{i} \in \mathbb{R}^{n}$ is the system state, $u_{i} \in \mathbb{R}^{m}$ is the input (or control), $y_{i} \in \mathbb{R}^{r}, A_{j} \in \mathbb{R}^{n \times n}\left(j \in \sigma_{0}^{p}\right)$ are the matrices of the state, $B_{j} \in \mathbb{R}^{n \times m}\left(j \in \sigma_{0}^{q}\right)$ are the matrices of the input, $C_{j} \in \mathbb{R}^{r \times n}\left(j \in \sigma_{0}^{l}\right)$ are the matrices of the output and $D_{j} \in \mathbb{R}^{r \times m}\left(j \in \sigma_{0}^{v}\right)$ are the matrices of the feedthrough (or feedforward), and $p, q$ and $v$, and $l$ are the nonnegative integer maximal values of delays on state, input, and output, respectively.

Definition 1. The system modeled by (2) and (3) is said to be positive if the state $x_{i} \in \mathbb{R}_{+}^{n}$ and the output $y_{i} \in \mathbb{R}_{+}^{r}, i \in \mathbb{N}$, for any initial states $x_{-j} \in \mathbb{R}_{+}^{n}\left(j \in \sigma_{0}^{p}\right)$ and for any initial inputs $u_{-j} \in \mathbb{R}_{+}^{m}\left(j \in \sigma_{1}^{q}\right)$ and all inputs $u_{i} \in \mathbb{R}_{+}^{m}, i \in \mathbb{N}$.

The mathematical theory of positive linear systems is based on the theory of nonnegative matrix developed by Perron and Frobenius (see $[16,30]$ ).

Definition 2. A matrix $A=\left(a_{i j}\right)$ in $\mathbb{R}^{n \times m}$ is said to be nonnegative and denoted by $A \in \mathbb{R}_{+}^{n \times m}$, if all of its elements are nonnegative; that is, $a_{i j} \geq 0$ for all $i \in \sigma_{1}^{n}, j \in \sigma_{1}^{m}$.

Remark 3. $A \in \mathbb{R}_{+}^{n \times m}$ if and only if $A x \in \mathbb{R}_{+}^{n}$ for all $x \in \mathbb{R}_{+}^{m}$. Indeed, suppose one of the elements of $A, a_{i j}$, is negative. Then, for the nonnegative vector $x=(0, \ldots, 0,1,0, \ldots, 0)^{T} \in$ $\mathbb{R}_{+}^{m}$ with the one in the $j$ th component, the $i$ th component of $A x$ would be $a_{i j}$, which is negative. It is also easy to verify the converse.

The following proposition provides a necessary and sufficient conditions for positivity of system (2) and (3).

Proposition 4. System (2) and (3) is positive if and only if

$$
\begin{array}{cc}
A_{j} \in \mathbb{R}_{+}^{n \times n} & \left(j \in \sigma_{0}^{p}\right), \\
B_{j} \in \mathbb{R}_{+}^{n \times m} & \left(j \in \sigma_{0}^{q}\right), \\
C_{j} \in \mathbb{R}_{+}^{r \times n} & \left(j \in \sigma_{0}^{l}\right), \\
D_{j} \in \mathbb{R}_{+}^{r \times m} & \left(j \in \sigma_{0}^{v}\right) .
\end{array}
$$

Proof.

Sufficiency. If the condition (4) is satisfied, then

$$
x_{1}=\sum_{j=0}^{p} A_{j} x_{-j}+\sum_{j=0}^{q} B_{j} u_{-j} \in \mathbb{R}_{+}^{n}
$$

since $x_{-j} \in \mathbb{R}_{+}^{n}\left(j \in \sigma_{0}^{p}\right)$ and $u_{-j} \in \mathbb{R}_{+}^{m}\left(j \in \sigma_{0}^{q}\right)$. Assume that $x_{k} \in \mathbb{R}_{+}^{n}$ for $k \in \sigma_{1}^{i}$. From (2) we have

$$
x_{i+1}=\sum_{j=0}^{p} A_{j} x_{i-j}+\sum_{j=0}^{q} B_{j} u_{i-j} \in \mathbb{R}_{+}^{n}
$$

since (4) holds and $x_{i-j} \in \mathbb{R}_{+}^{n}\left(j \in \sigma_{0}^{p}\right), u_{-j} \in \mathbb{R}_{+}^{m}\left(j \in \sigma_{1}^{q}\right)$, and $u_{i} \in \mathbb{R}_{+}^{m}, i \in \mathbb{N}$. Hence $x_{i} \in \mathbb{R}_{+}^{n}$ for any $i \in \mathbb{N}$. 
Consequently, if condition (5) is satisfied, we get that $y_{i} \in \mathbb{R}_{+}^{r}$ for every $i \in \mathbb{N}$ since $x_{-j} \in \mathbb{R}_{+}^{n}\left(j \in \sigma_{0}^{l}\right), u_{-j} \in \mathbb{R}_{+}^{m}\left(j \in \sigma_{1}^{v}\right)$, and $u_{i} \in \mathbb{R}_{+}^{m}, i \in \mathbb{N}$.

Necessity. Assuming that system (2) and (3) is positive, let $u_{-j}=0$ for $j \in \sigma_{0}^{q}$. Then from (2) and (3), for $i=0$, we have

$$
\begin{aligned}
& x_{1}=\sum_{j=0}^{p} A_{j} x_{-j}=\bar{A} \bar{x}_{0} \in \mathbb{R}_{+}^{n}, \\
& y_{0}=\sum_{j=0}^{l} C_{j} x_{-j}=\bar{C} \bar{x}_{1} \in \mathbb{R}_{+}^{r},
\end{aligned}
$$

with

$$
\begin{aligned}
& \bar{A}=\left(\begin{array}{llll}
A_{0} & A_{1} & \cdots & A_{p}
\end{array}\right) \in \mathbb{R}^{n \times n(p+1)}, \\
& \bar{C}=\left(\begin{array}{llll}
C_{0} & C_{1} & \cdots & C_{l}
\end{array}\right) \in \mathbb{R}^{r \times n(l+1)}, \\
& \bar{x}_{0}=\left(x_{0}, x_{-1}, \ldots, x_{-p}\right)^{T} \in \mathbb{R}^{n(p+1)} \text {, } \\
& \bar{x}_{1}=\left(x_{0}, x_{-1}, \ldots, x_{-l}\right)^{T} \in \mathbb{R}^{n(l+1)} \text {. }
\end{aligned}
$$

Hence by Remark 3, we have $\bar{A} \in \mathbb{R}_{+}^{n \times n(p+1)}$; that is, $A_{j} \in$ $\mathbb{R}_{+}^{n \times n}\left(j \in \sigma_{0}^{p}\right)$ and $\bar{C} \in \mathbb{R}_{+}^{r \times n(l+1)}$; that is, $C_{j} \in \mathbb{R}_{+}^{r \times n}\left(j \in \sigma_{0}^{l}\right)$ since $\bar{x}_{0} \in \mathbb{R}_{+}^{n(p+1)}$ and $\bar{x}_{1} \in \mathbb{R}_{+}^{n(l+1)}$ are arbitrary. Now, assume that $x_{-j}=0$ for $j \in \sigma_{0}^{p}$, and for $i=0$, we obtain

$$
\begin{aligned}
& x_{1}=\sum_{j=0}^{q} B_{j} u_{-j}=\bar{B} \bar{u}_{0} \in \mathbb{R}_{+}^{n}, \\
& y_{0}=\sum_{j=0}^{v} D_{j} u_{-j}=\bar{D} \bar{u}_{1} \in \mathbb{R}_{+}^{r},
\end{aligned}
$$

with

$$
\begin{aligned}
& \bar{B}=\left(\begin{array}{llll}
B_{0} & B_{1} & \cdots & B_{q}
\end{array}\right) \in \mathbb{R}^{n \times m(q+1)}, \\
& \bar{D}=\left(\begin{array}{llll}
D_{0} & D_{1} & \cdots & D_{v}
\end{array}\right) \in \mathbb{R}^{r \times m(v+1)}, \\
& \bar{u}_{0}=\left(u_{0}, u_{-1}, \ldots, u_{-q}\right)^{T} \in \mathbb{R}^{m(q+1)}, \\
& \bar{u}_{1}=\left(u_{0}, u_{-1}, \ldots, u_{-v}\right)^{T} \in \mathbb{R}^{m(v+1)} \text {, }
\end{aligned}
$$

which implies that $\bar{B} \in \mathbb{R}_{+}^{n \times m(q+1)}$; that is, $B_{j} \in \mathbb{R}_{+}^{n \times m}\left(j \in \sigma_{0}^{q}\right)$ and $\bar{D} \in \mathbb{R}_{+}^{r \times m(v+1)}$, that is, $D_{j} \in \mathbb{R}_{+}^{r \times m}\left(j \in \sigma_{0}^{v}\right)$ since $\bar{u}_{0} \in$ $\mathbb{R}_{+}^{m(q+1)}$ and $\bar{u}_{1} \in \mathbb{R}_{+}^{m(v+1)}$ are arbitrary. This completes the proof.

In all the sequel, we assume that system (2) and (3) is positive.

In the next proposition, we will present the explicit solution of system (2).
Proposition 5. The general solution to (2) is given by

$$
\begin{aligned}
& x_{i}= G_{i} x_{0}+\sum_{j=1}^{p} \sum_{k=1}^{p-j+1} G_{i-k} A_{k-1+j} x_{-j} \\
&+ \sum_{j=1}^{q} \sum_{k=1}^{q-j+1} G_{i-k} B_{k-1+j} u_{-j}+\sum_{j=0}^{i-1} \sum_{k=0}^{q} G_{i-1-j-k} B_{k} u_{j}, \\
& i \in \mathbb{N},
\end{aligned}
$$

where the transition matrix $G_{i} \in \mathbb{R}^{n \times n}(i \in \mathbb{N})$ is determined by the recurrence relation

$$
G_{i}= \begin{cases}I_{n} & \text { for } i=0, \\ \sum_{k=0}^{p} A_{k} G_{i-1-k} & \text { for } i \in \mathbb{N}_{+}\end{cases}
$$

with the assumption

$$
G_{i}=0 \quad \text { for } i<0 .
$$

Proof. The proof is given in [31].

We pose $H_{i}^{0}=G_{i}$, and then

$$
H_{i}^{0}= \begin{cases}I_{n} & \text { for } i=0 \\ \sum_{k=0}^{p} A_{k} H_{i-1-k}^{0} & \text { for } i \in \mathbb{N}_{+} \\ 0 & \text { for } i<0\end{cases}
$$

and, for all $i \in \mathbb{N}_{+}$, we pose

$$
\begin{gathered}
H_{i}^{j}=\sum_{k=1}^{p-j+1} H_{i-k}^{0} A_{k-1+j}, \quad j \in \sigma_{1}^{p}, \\
L_{i}^{j}=\sum_{k=1}^{q-j+1} H_{i-k}^{0} B_{k-1+j}, \quad j \in \sigma_{1}^{q},
\end{gathered}
$$

with $H_{i}^{j}=L_{i}^{j}=0$ for $i \leq 0$.

Moreover, for $i \in \mathbb{N}$, we pose

$$
K_{i}=\sum_{k=0}^{q} H_{i-k}^{0} B_{k},
$$

with $K_{i}=0$, for $i<0$.

Clearly by (15), (16), and (17), the solution of (2) is given by the following new formula:

$$
x_{i}=H_{i}^{0} x_{0}+\sum_{j=1}^{p} H_{i}^{j} x_{-j}+\sum_{j=1}^{q} L_{i}^{j} u_{-j}+\sum_{j=0}^{i-1} K_{i-1-j} u_{j},
$$

$i \in \mathbb{N}$.

In the following and without loss of generality, we assume that $l=v$. Indeed, for example, if $l>v$ we can set $D_{j}=0$ for $j \in \sigma_{v+1}^{l}$. 
Now, we introduce the matrices sequence as follows:

$$
\begin{aligned}
& \mathscr{H}_{i}^{j}=\sum_{k=0}^{l} C_{k} H_{i-k}^{j}, \quad j \in \sigma_{0}^{p}, \quad i \in \mathbb{N}, \\
& \mathscr{L}_{i}^{j}=\sum_{k=0}^{l} C_{k} L_{i-k}^{j}, \quad j \in \sigma_{1}^{q}, \quad i \in \mathbb{N}, \\
& \mathscr{K}_{i}=\sum_{k=0}^{l} C_{k} K_{i-k}, \quad i \in \mathbb{N}, \\
& \mathscr{\mathscr { K }}_{i}=\mathscr{K}_{i}+D_{i+1}, \quad i \in \sigma_{0}^{l-1} .
\end{aligned}
$$

For $0 \leq i<l$, the output equation (3) can be rewritten as

$$
\begin{aligned}
& y_{i}=\sum_{k=0}^{i} C_{k} x_{i-k}+\sum_{k=i+1}^{l} C_{k} x_{i-k}+\sum_{k=0}^{i} D_{k} u_{i-k} \\
& +\sum_{k=i+1}^{l} D_{k} u_{i-k} \\
& =\sum_{k=0}^{i} C_{k} H_{i-k}^{0} x_{0}+\sum_{k=0}^{i} C_{k} \sum_{j=1}^{p} H_{i-k}^{j} x_{-j} \\
& +\sum_{k=0}^{i} C_{k} \sum_{j=1}^{q} L_{i-k}^{j} u_{-j}+\sum_{k=i+1}^{l} C_{k} x_{i-k}+\sum_{k=i+1}^{l} D_{k} u_{i-k} \\
& +\sum_{k=0}^{i} C_{k} \sum_{j=0}^{i-k-1} K_{i-k-1-j} u_{j}+\sum_{k=0}^{i} D_{k} u_{i-k} \\
& =\sum_{k=0}^{l} C_{k} H_{i-k}^{0} x_{0}+\sum_{k=0}^{l} C_{k} \sum_{j=1}^{p} H_{i-k}^{j} x_{-j} \\
& +\sum_{k=0}^{l} C_{k} \sum_{j=1}^{q} L_{i-k}^{j} u_{-j}+\sum_{k=i+1}^{l} C_{k} x_{i-k}+\sum_{k=i+1}^{l} D_{k} u_{i-k} \\
& +\sum_{k=0}^{i-1} C_{k} \sum_{j=0}^{i-k-1} K_{i-k-1-j} u_{j}+\sum_{k=0}^{i} D_{k} u_{i-k} \\
& =\left(\sum_{k=0}^{l} C_{k} H_{i-k}^{0}\right) x_{0}+\sum_{j=1}^{p}\left(\sum_{k=0}^{l} C_{k} H_{i-k}^{j}\right) x_{-j} \\
& +\sum_{j=1}^{q}\left(\sum_{k=0}^{l} C_{k} L_{i-k}^{j}\right) u_{-j}+\sum_{j=i+1}^{l} C_{j} x_{i-j} \\
& +\sum_{j=i+1}^{l} D_{j} u_{i-j}+\sum_{j=0}^{i-1}\left(\sum_{k=0}^{i-j-1} C_{k} K_{i-j-1-k}\right) u_{j} \\
& +\sum_{j=0}^{i-1} D_{i-j} u_{j}+D_{0} u_{i}
\end{aligned}
$$

$$
\begin{aligned}
= & \mathscr{H}_{i}^{0} x_{0}+\sum_{j=1}^{p} \mathscr{H}_{i}^{j} x_{-j}+\sum_{j=1}^{q} \mathscr{L}_{i}^{j} u_{-j}+\sum_{j=1}^{l-i} C_{i+j} x_{-j} \\
& +\sum_{j=1}^{l-i} D_{i+j} u_{-j}+\sum_{j=0}^{i-1}\left(\mathscr{K}_{i-j-1}+D_{i-j}\right) u_{j}+D_{0} u_{i} \\
= & \mathscr{H}_{i}^{0} x_{0}+\sum_{j=1}^{l-i}\left(\mathscr{H}_{i}^{j}+C_{i+j}\right) x_{-j}+\sum_{j=l-i+1}^{p} \mathscr{H}_{i}^{j} x_{-j} \\
& +\sum_{j=1}^{l-i}\left(\mathscr{L}_{i}^{j}+D_{i+j}\right) u_{-j}+\sum_{j=l-i+1}^{q} \mathscr{L}_{i}^{j} u_{-j} \\
& +\sum_{j=0}^{i-1} \mathscr{K}_{i-j-1} u_{j}+D_{0} u_{i} .
\end{aligned}
$$

Hence

$$
y_{i}=Q_{i+1} \tilde{x}_{0}+\mathscr{R}_{i+1} u_{0}^{i+1},
$$

with

$$
Q_{i+1}=\left(\begin{array}{ll}
M_{i+1} & O_{i+1}
\end{array}\right) \in \mathbb{R}_{+}^{r \times(n(p+1)+m q)},
$$

where

$$
\begin{aligned}
& M_{i+1}
\end{aligned}
$$

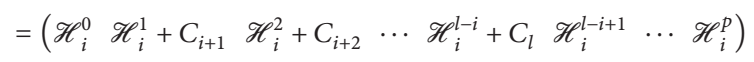

$$
\begin{aligned}
& \in \mathbb{R}_{+}^{r \times n(p+1)} \\
& O_{i+1}=\left(\begin{array}{llllll}
\mathscr{L}_{i}^{1}+D_{i+1} & \cdots & \mathscr{L}_{i}^{l-i}+D_{l} & \mathscr{L}_{i}^{l-i+1} & \cdots & \mathscr{L}_{i}^{q}
\end{array}\right) \in \mathbb{R}_{+}^{r \times m q}, \\
& \tilde{x}_{0}=\left(\begin{array}{c}
x_{0} \\
x_{-1} \\
\vdots \\
x_{-p} \\
u_{-1} \\
\vdots \\
u_{-q}
\end{array}\right) \in \mathbb{R}_{+}^{n(p+1)+m q} \\
& \mathscr{R}_{i+1}=\left(\begin{array}{llllll}
\overline{\mathscr{K}}_{i-1} & \overline{\mathscr{K}}_{i-2} & \cdots & \overline{\mathscr{K}}_{1} & \overline{\mathscr{K}}_{0} & D_{0}
\end{array}\right) \in \mathbb{R}_{+}^{r \times(i+1) m}, \\
& u_{0}^{i+1}=\left(\begin{array}{c}
u_{0} \\
u_{1} \\
\vdots \\
u_{i}
\end{array}\right) \in \mathbb{R}_{+}^{(i+1) m} .
\end{aligned}
$$


For $i \geq l$, we have

$$
\begin{aligned}
& y_{i}=\sum_{k=0}^{l} C_{k} H_{i-k}^{0} x_{0}+\sum_{k=0}^{l} C_{k} \sum_{j=1}^{p} H_{i-k}^{j} x_{-j} \\
& +\sum_{k=0}^{l} C_{k} \sum_{j=1}^{q} L_{i-k}^{j} u_{-j}+\sum_{k=0}^{l} C_{k} \sum_{j=0}^{i-k-1} K_{i-k-1-j} u_{j} \\
& +\sum_{k=0}^{l} D_{k} u_{i-k} \\
& =\left(\sum_{k=0}^{l} C_{k} H_{i-k}^{0}\right) x_{0}+\sum_{j=1}^{p}\left(\sum_{k=0}^{l} C_{k} H_{i-k}^{j}\right) x_{-j} \\
& +\sum_{j=1}^{q}\left(\sum_{k=0}^{l} C_{k} L_{i-k}^{j}\right) u_{-j} \\
& +\sum_{j=0}^{i-l-1}\left(\sum_{k=0}^{l} C_{k} K_{i-j-1-k}\right) u_{j} \\
& +\sum_{j=i-l}^{i-1}\left(\sum_{k=0}^{i-j-1} C_{k} K_{i-j-1-k}\right) u_{j}+\sum_{j=0}^{l} D_{j} u_{i-j} \\
& =\left(\sum_{k=0}^{l} C_{k} H_{i-k}^{0}\right) x_{0}+\sum_{j=1}^{p}\left(\sum_{k=0}^{l} C_{k} H_{i-k}^{j}\right) x_{-j} \\
& +\sum_{j=1}^{q}\left(\sum_{k=0}^{l} C_{k} L_{i-k}^{j}\right) u_{-j} \\
& +\sum_{j=0}^{i-1}\left(\sum_{k=0}^{l} C_{k} K_{i-j-1-k}\right) u_{j}+\sum_{j=0}^{l} D_{j} u_{i-j} \\
& =\mathscr{H}_{i}^{0} x_{0}+\sum_{j=1}^{p} \mathscr{H}_{i}^{j} x_{-j}+\sum_{j=1}^{q} \mathscr{L}_{i}^{j} u_{-j}+\sum_{j=0}^{i-1} \mathscr{K}_{i-j-1} u_{j} \\
& +\sum_{j=0}^{l} D_{j} u_{i-j} \\
& =\mathscr{H}_{i}^{0} x_{0}+\sum_{j=1}^{p} \mathscr{H}_{i}^{j} x_{-j}+\sum_{j=1}^{q} \mathscr{L}_{i}^{j} u_{-j}+\sum_{j=0}^{i-l-1} \mathscr{K}_{i-j-1} u_{j} \\
& +\sum_{j=i-l}^{i-1}\left(\mathscr{K}_{i-j-1}+D_{i-j}\right) u_{j}+D_{0} u_{i} \\
& =\mathscr{H}_{i}^{0} x_{0}+\sum_{j=1}^{p} \mathscr{H}_{i}^{j} x_{-j}+\sum_{j=1}^{p} \mathscr{L}_{i}^{j} u_{-j}+\sum_{j=0}^{i-l-1} \mathscr{K}_{i-j-1} u_{j} \\
& +\sum_{j=i-l}^{i-1} \overline{\mathscr{K}}_{i-j-1} u_{j}+D_{0} u_{i}
\end{aligned}
$$

Then, we get the linear algebraic equation

$$
y_{i}=Q_{i+1} \tilde{x}_{0}+\mathscr{R}_{i+1} u_{0}^{i+1} \text {, }
$$

with

$$
\begin{aligned}
& Q_{i+1}=\left(\begin{array}{lllllll}
\mathscr{H}_{i}^{0} & \mathscr{H}_{i}^{1} & \cdots & \mathscr{H}_{i}^{p} & \mathscr{L}_{i}^{1} & \cdots & \mathscr{L}_{i}^{q}
\end{array}\right) \\
& \in \mathbb{R}_{+}^{r \times(n(p+1)+m q)},
\end{aligned}
$$

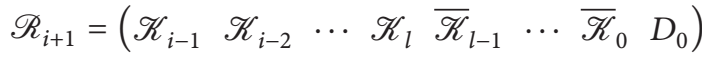

$$
\begin{aligned}
& \in \mathbb{R}_{+}^{r \times(i+1) m} \text {. }
\end{aligned}
$$

The following lemmas will be needed in the sequel.

Lemma 6. For any $i \in \mathbb{N}_{+}$, we have

$$
H_{i}^{0}=\sum_{k=0}^{p} H_{i-1-k}^{0} A_{k} .
$$

Proof. First, for $i=1$, we have $H_{1}^{0}=A_{0}=\sum_{k=0}^{p} H_{-k}^{0} A_{k}$ and (28) holds. Secondly, suppose that (28) holds for $k \in \sigma_{1}^{i}$. We prove that it holds for $k=i+1$.

For $i \in \sigma_{1}^{p}$, we have

$$
\begin{aligned}
H_{i+1}^{0} & =\sum_{k=0}^{i} A_{k} H_{i-k}^{0}=\sum_{k=0}^{i-1} A_{k} H_{i-k}^{0}+A_{i} \\
& =\sum_{k=0}^{i-1} A_{k}\left(\sum_{j=0}^{p} H_{i-k-1-j}^{0} A_{j}\right)+A_{i} \\
& =\sum_{k=0}^{i-1} A_{k}\left(\sum_{j=0}^{i-k-1} H_{i-k-1-j}^{0} A_{j}\right)+A_{i} \\
& =\sum_{j=0}^{i-1}\left(\sum_{k=0}^{i-j-1} A_{k} H_{i-j-1-k}^{0}\right) A_{j}+A_{i} \\
& =\sum_{j=0}^{i-1} H_{i-j}^{0} A_{j}+A_{i}=\sum_{j=0}^{i} H_{i-j}^{0} A_{j}=\sum_{j=0}^{p} H_{i-j}^{0} A_{j} .
\end{aligned}
$$

For $i \geq p+1$, we have

$$
\begin{aligned}
H_{i+1}^{0} & =\sum_{k=0}^{p} A_{k} H_{i-k}^{0}=\sum_{k=0}^{p} A_{k}\left(\sum_{j=0}^{p} H_{i-k-1-j}^{0} A_{j}\right) \\
& =\sum_{j=0}^{p}\left(\sum_{k=0}^{p} A_{k} H_{i-j-1-k}^{0}\right) A_{j}=\sum_{j=0}^{p} H_{i-j}^{0} A_{j} .
\end{aligned}
$$

Thus, (28) is satisfied in step $i+1$. Hence, (28) holds for any $i \in \mathbb{N}_{+}$.

Lemma 7. For all $i \in \mathbb{N}$, we have

$$
\begin{aligned}
& H_{i+1}^{j}=H_{i}^{j+1}+H_{i}^{0} A_{j}, \quad j \in \sigma_{0}^{p-1}, \\
& H_{i+1}^{p}=H_{i}^{0} A_{p},
\end{aligned}
$$




$$
\begin{aligned}
& L_{i+1}^{j}=L_{i}^{j+1}+H_{i}^{0} B_{j}, \quad j \in \sigma_{1}^{q-1}, \\
& L_{i+1}^{q}=H_{i}^{0} B_{q} .
\end{aligned}
$$

Proof. For $i=0$, we have

$$
\begin{gathered}
H_{0}^{j+1}+H_{0}^{0} A_{j}=A_{j}=H_{1}^{j}, \quad j \in \sigma_{0}^{p-1}, \\
H_{0}^{0} A_{p}=A_{p}=H_{1}^{p} .
\end{gathered}
$$

Let $i \in \mathbb{N}_{+}$. For $j=0$, we have

$$
H_{i}^{1}+H_{i}^{0} A_{0}=\sum_{k=1}^{p} H_{i-k}^{0} A_{k}+H_{i}^{0} A_{0}=\sum_{k=0}^{p} H_{i-k}^{0} A_{k} ;
$$

then by Lemma 6 , we get

$$
H_{i+1}^{0}=H_{i}^{1}+H_{i}^{0} A_{0} .
$$

For $j \in \sigma_{1}^{p-1}$, we have

$$
\begin{aligned}
H_{i+1}^{j}-H_{i}^{j+1} & =\sum_{k=1}^{p-j+1} H_{i+1-k}^{0} A_{k-1+j}-\sum_{k=1}^{p-j} H_{i-k}^{0} A_{k+j} \\
& =\sum_{k=0}^{p-j} H_{i-k}^{0} A_{k+j}-\sum_{k=1}^{p-j} H_{i-k}^{0} A_{k+j}=H_{i}^{0} A_{j} .
\end{aligned}
$$

And for $j=p$, we have

$$
H_{i+1}^{p}=\sum_{k=1}^{1} H_{i+1-k}^{0} A_{k-1+p}=H_{i}^{0} A_{p} .
$$

Similarly, we prove that (32) holds.

Lemma 8. We have

$$
\begin{aligned}
& \mathscr{H}_{i+1}^{0}=\mathscr{H}_{i}^{1}+\mathscr{H}_{i}^{0} A_{0}+C_{i+1}, \quad i<l, \\
& \mathscr{H}_{i+1}^{0}=\mathscr{H}_{i}^{1}+\mathscr{H}_{i}^{0} A_{0}, \quad i \geq l .
\end{aligned}
$$

And for all $i \in \mathbb{N}$, we have

$$
\begin{aligned}
& \mathscr{H}_{i+1}^{j}=\mathscr{H}_{i}^{j+1}+\mathscr{H}_{i}^{0} A_{j}, \quad j \in \sigma_{1}^{p-1}, \\
& \mathscr{H}_{i+1}^{p}=\mathscr{H}_{i}^{0} A_{p}, \\
& \mathscr{L}_{i+1}^{j}=\mathscr{L}_{i}^{j+1}+\mathscr{H}_{i}^{0} B_{j}, \quad j \in \sigma_{1}^{q-1}, \\
& \mathscr{L}_{i+1}^{q}=\mathscr{H}_{i}^{0} B_{q} .
\end{aligned}
$$

Proof. Let $i<l$. For $j=0$, we have

$$
\begin{aligned}
\mathscr{H}_{i+1}^{0} & =\sum_{k=0}^{l} C_{k} H_{i+1-k}^{0}=\sum_{k=0}^{i+1} C_{k} H_{i+1-k}^{0} \\
& =\sum_{k=0}^{i} C_{k} H_{i+1-k}^{0}+C_{i+1} \\
& =\sum_{k=0}^{i} C_{k}\left(H_{i-k}^{1}+H_{i-k}^{0} A_{0}\right)+C_{i+1} \\
& =\sum_{k=0}^{i} C_{k} H_{i-k}^{1}+\left(\sum_{k=0}^{i} C_{k} H_{i-k}^{0}\right) A_{0}+C_{i+1} \\
& =\mathscr{H}_{i}^{1}+\mathscr{H}_{i}^{0} A_{0}+C_{i+1},
\end{aligned}
$$

for $j \in \sigma_{1}^{p-1}$, we have

$$
\begin{aligned}
\mathscr{H}_{i+1}^{j} & =\sum_{k=0}^{l} C_{k} H_{i+1-k}^{j}=\sum_{k=0}^{i} C_{k} H_{i+1-k}^{j} \\
& =\sum_{k=0}^{i} C_{k}\left(H_{i-k}^{j+1}+H_{i-k}^{0} A_{j}\right)=\mathscr{H}_{i}^{j+1}+\mathscr{H}_{i}^{0} A_{j},
\end{aligned}
$$

and, for $j=p$, we have

$$
\mathscr{H}_{i+1}^{p}=\sum_{k=0}^{i} C_{k} H_{i+1-k}^{p}=\left(\sum_{k=0}^{i} C_{k} H_{i-k}^{0}\right) A_{p}=\mathscr{H}_{i}^{0} A_{p} .
$$

For $i \geq l$, with $j \in \sigma_{0}^{p-1}$, we have

$$
\begin{aligned}
\mathscr{H}_{i+1}^{j} & =\sum_{k=0}^{l} C_{k} H_{i+1-k}^{j}=\sum_{k=0}^{l} C_{k}\left(H_{i-k}^{j+1}+H_{i-k}^{0} A_{j}\right) \\
& =\mathscr{H}_{i}^{j+1}+\mathscr{H}_{i}^{0} A_{j},
\end{aligned}
$$

and, for $j=p$, we have

$$
\mathscr{H}_{i+1}^{p}=\sum_{k=0}^{l} C_{k} H_{i+1-k}^{p}=\left(\sum_{k=0}^{l} C_{k} H_{i-k}^{0}\right) A_{p}=\mathscr{H}_{i}^{0} A_{p} .
$$

Similarly, we prove that (40) holds.

\section{Output Reachability}

In this section we will present necessary and sufficient conditions for output reachability of system (2) and (3). By generalization of definition given in [29] we obtain the following definitions.

Definition 9. The system modeled by (2) and (3) is said to be output reachable in $N \in \mathbb{N}_{+}$steps if, for any nonnegative final output $y_{f} \in \mathbb{R}_{+}^{r}$, there exists a nonnegative input sequence $u_{i} \in \mathbb{R}_{+}^{m}, i \in \sigma_{0}^{N-1}$, which steers the output of the system from $x_{-j}=0, j \in \sigma_{0}^{p}$ to $y_{f}$, with $u_{-j}=0$ for $j \in \sigma_{1}^{p}$; that is, $y_{f}=y_{N-1}$ 
Definition 10. The system modeled by (2) and (3) is said to be output reachable if there exists a positive integer $N \in \mathbb{N}_{+}$such that the system is output reachable in $N$ steps.

Now, we present a class of nonnegative matrices, called the monomial matrices $[18,30]$. The utility of such a matrix will be highlighted in the study of the output reachability of positive linear systems.

A vector $v \in \mathbb{R}_{+}^{n}$ with exactly one of its components being nonzero and all the others being zero is called monomial vector or $i$-monomial if the nonzero component is in the $i$ th position.

Definition 11. A square matrix $A \in \mathbb{R}_{+}^{n \times n}$ is said to be monomial if it contains $n$ linearly independent monomial columns.

An important property of monomial matrices is given by the following result.

Lemma 12 (see [18]). Let $A \in \mathbb{R}_{+}^{n \times n}$. Then $A^{-1}$ exists and is nonnegative if and only if $A$ is a monomial matrix. Furthermore, $A^{-1}$ is also a monomial matrix.

The characterization of the output reachability is given by the following proposition.

Proposition 13. The system modeled by (2) and (3) is output reachable if and only if, for some $N \in \mathbb{N}_{+}$, the output reachability matrix $\mathscr{R}_{N}$ includes a monomial submatrix of order $r \times r(r \leq N m)$.

Proof.

Sufficiency. Let $y_{f} \in \mathbb{R}_{+}^{r}$ be the final output to be reached. From (21) or (25), we have

$$
y_{N-1}=Q_{N} \tilde{x}_{0}+\mathscr{R}_{N} u_{0}^{N} .
$$

With $\tilde{x}_{0}=0$, this gives

$$
y_{N-1}=\mathscr{R}_{N} u_{0}^{N}
$$

The matrix $\mathscr{R}_{N}$ includes a monomial submatrix of order $r \times r$, and without loss of generality, we can assume that

$$
\mathscr{R}_{N}=\left(\begin{array}{ll}
R_{1} & R_{2}
\end{array}\right)
$$

such that $R_{1} \in \mathbb{R}_{+}^{r \times r}$ is a monomial matrix and $R_{2} \in$ $\mathbb{R}_{+}^{r \times(N m-r)}$. Hence, by Lemma 12 , we have $R_{1}^{-1} \in \mathbb{R}_{+}^{r \times r}$. Thus, for

$$
u_{0}^{N}=\left(\begin{array}{c}
R_{1}^{-1} y_{f} \\
0
\end{array}\right) \in \mathbb{R}_{+}^{N m}
$$

we get

$$
y_{N-1}=\left(\begin{array}{ll}
R_{1} & R_{2}
\end{array}\right)\left(\begin{array}{c}
R_{1}^{-1} y_{f} \\
0
\end{array}\right)=y_{f} \text {; }
$$

that is, system (2) and (3) is output reachable.
Necessity. Assume that system (2) and (3) is output reachable for some $N \in \mathbb{N}_{+}$. Thus, for every $z \in \mathbb{R}_{+}^{r}$ there exists an input $u^{N} \in \mathbb{R}_{+}^{N m}$ such that

$$
z=\mathscr{R}_{N} u^{N}
$$

with $\mathscr{R}_{N}=\left(r_{i j}\right)_{i \in \sigma_{1}^{r}, j \in \sigma_{1}^{N m}}$ and $u^{N}=\left(u_{j}\right)_{j \in \sigma_{1}^{N m}}$. In particular, for $z=e_{1}$, with $e_{1}$ being the first column of $I_{r}$, we have

$$
\sum_{j=1}^{N m} r_{1 j} u_{j}=1
$$

and for $i \in \sigma_{2}^{r}$, we have

$$
\sum_{j=1}^{N m} r_{i j} u_{j}=0
$$

So by (52), there exists $k \in \sigma_{1}^{N m}$ such that $u_{k} \neq 0$, and consequently by equation (53) we have $r_{i k}=0$ for all $i \in \sigma_{2}^{r}$. Hence, if $r_{1 k} \neq 0$, then the $k$ th column of $\mathscr{R}_{N}$ is monomial. If $r_{1 k}=0$, then the $k$ th column of $\mathscr{R}_{N}$ is null, which implies that

$$
\begin{aligned}
& \sum_{j=1}^{N m} r_{1 j} u_{j}=1, \quad j \neq k, \\
& \sum_{j=1}^{N m} r_{i j} u_{j}=0, \quad j \neq k, \quad i \in \sigma_{2}^{r} .
\end{aligned}
$$

The same reasoning gives the existence of a 1-monomial column or another null column of $\mathscr{R}_{N}$. Since the columns of $\mathscr{R}_{N}$ are not all null, then $\mathscr{R}_{N}$ has at least one 1-monomial column.

The same reasoning for $z=e_{i}, i \in \sigma_{2}^{r}$, leads to the existence of a $i$-monomial column. Hence by Definition 11, the matrix $\mathscr{R}_{N}$ contains a monomial submatrix of order $r \times r$. The proposition is proved.

Remark 14. If system (2) and (3) is output reachable and

$$
\mathscr{R}_{N}^{T}\left(\mathscr{R}_{N} \mathscr{R}_{N}^{T}\right)^{-1} \in \mathbb{R}_{+}^{N m \times r},
$$

then the nonnegative input $u_{0}^{N} \in \mathbb{R}_{+}^{N m}$ which steers the output of the system from $x_{-j}=0, j \in \sigma_{0}^{p}$, to any desired nonnegative final output $y_{f} \in \mathbb{R}_{+}^{r}$, with $u_{-j}=0$ for $j \in \sigma_{1}^{q}$, can be computed by the formula

$$
u_{0}^{N}=\mathscr{R}_{N}^{T}\left(\mathscr{R}_{N} \mathscr{R}_{N}^{T}\right)^{-1} y_{f} .
$$

\section{Null Output Controllability}

By generalization of definition given in [11] the precise definitions of the null output controllability of system (2) and (3) are given as follows.

Definition 15. The system modeled by (2) and (3) is said to be null output controllable in $N \in \mathbb{N}_{+}$steps if, for any 
nonnegative initial state sequence $x_{-j} \in \mathbb{R}_{+}^{n}\left(j \in \sigma_{0}^{p}\right)$ and any nonnegative initial input sequence $u_{-j} \in \mathbb{R}_{+}^{m}\left(j \in \sigma_{1}^{q}\right)$, there exists a nonnegative input sequence $u_{i} \in \mathbb{R}_{+}^{m}, i \in \sigma_{0}^{N-1}$, which steers the output of the system from $x_{-j}$ to zero; that is, $y_{N-1}=0$.

Definition 16. The system modeled by (2) and (3) is said to be null output controllable if there exists a positive integer $N \in \mathbb{N}_{+}$such that the system is null output controllable in $N$ steps.

The characterization of the null output controllability is given by the following proposition.

Proposition 17. The system modeled by (2) and (3) is null output controllable if and only if, for some $N \in \mathbb{N}_{+}$, the null output controllability matrix $\mathbb{Q}_{N}$ is null.

Proof.

Sufficiency. From (21) or (25), at the step $i=N-1$, we have

$$
y_{N-1}=\mathscr{Q}_{N} \tilde{x}_{0}+\mathscr{R}_{N} u_{0}^{N} ;
$$

since $\mathbb{Q}_{N}=0$, then, for $u_{0}^{N}=0$, we have $y_{N-1}=0$; that is, system (2) and (3) is null output controllable.

Necessity. If system (2) and (3) is null output controllable, then, for some $N \in \mathbb{N}_{+}$, there exists an input $u_{0}^{N} \in \mathbb{R}_{+}^{N m}$ such that

$$
Q_{N} \widetilde{x}_{0}+\mathscr{R}_{N} u_{0}^{N}=0 .
$$

Since $\mathscr{R}_{N} u_{0}^{N} \in \mathbb{R}_{+}^{r}$ and $\mathscr{Q}_{N} \tilde{x}_{0} \in \mathbb{R}_{+}^{r}$, then $\mathscr{Q}_{N} \widetilde{x}_{0}=0$, which ensures that $\mathscr{Q}_{N}=0$ because $\tilde{x}_{0} \in \mathbb{R}_{+}^{n(p+1)+m q}$ by Definition 15, is arbitrary. This finishes the proof.

System (2) and (3) describes the evolution of the state and output of a system in the nonnegative orthant with delays in the state, input, and output. However, we can rewrite this system in such a way that these delays disappear from the state equation. Let $\left(x_{i}\right)_{i \in \mathbb{N}}$ be the solution of (2) and define a new state variable $\widetilde{x}_{i} \in \mathbb{R}_{+}^{n(p+1)+m q}$ for $i \in \mathbb{N}$ by

$$
\tilde{x}_{i}=\left(\begin{array}{c}
x_{i} \\
x_{i-1} \\
\vdots \\
x_{i-p} \\
u_{i-1} \\
\vdots \\
u_{i-q}
\end{array}\right) .
$$

It is readily verified that the state $\widetilde{x}_{i}$ satisfies

$$
\begin{aligned}
\tilde{x}_{i+1} & =A \tilde{x}_{i}+B u_{i}, \quad i \in \mathbb{N}, \\
\tilde{x}_{0} & \in \mathbb{R}_{+}^{n(p+1)+m q},
\end{aligned}
$$

and the output $y_{i}$ satisfies

$$
y_{i}=C \widetilde{x}_{i}+D_{0} u_{i}, \quad i \in \mathbb{N},
$$

where

A

$$
=\left(\begin{array}{cccccccccc}
A_{0} & A_{1} & \cdots & \cdots & A_{p} & B_{1} & \cdots & \cdots & \cdots & B_{q} \\
I_{n} & 0 & \cdots & \cdots & 0 & 0 & \cdots & \cdots & \cdots & 0 \\
0 & \ddots & \ddots & & \vdots & \vdots & & & & \vdots \\
\vdots & \ddots & \ddots & \ddots & \vdots & \vdots & & & & \vdots \\
0 & \cdots & 0 & I_{n} & 0 & 0 & \cdots & \cdots & \cdots & 0 \\
0 & \cdots & \cdots & \cdots & 0 & 0 & \cdots & \cdots & \cdots & 0 \\
\vdots & & & & \vdots & I_{m} & \ddots & & & \vdots \\
\vdots & & & & \vdots & 0 & \ddots & \ddots & & \vdots \\
\vdots & & & & \vdots & \vdots & \ddots & \ddots & \ddots & \vdots \\
0 & \cdots & \cdots & \cdots & 0 & 0 & \cdots & 0 & I_{m} & 0
\end{array}\right),
$$

$$
B=\left(\begin{array}{c}
B_{0} \\
0 \\
\vdots \\
0 \\
I_{m} \\
0 \\
\vdots \\
0
\end{array}\right) \in \mathbb{R}_{+}^{(n(p+1)+m q) \times m},
$$

$$
C=\left(\begin{array}{ll}
C_{11} & C_{12}
\end{array}\right),
$$

where

$$
\begin{aligned}
& C_{11}=\left(\begin{array}{lllllll}
C_{0} & C_{1} & \cdots & C_{l} & 0 & \cdots & 0
\end{array}\right) \in \mathbb{R}_{+}^{r \times n(p+1)}, \\
& C_{12}=\left(\begin{array}{llllll}
D_{1} & \cdots & D_{l} & 0 & \cdots & 0
\end{array}\right) \in \mathbb{R}_{+}^{r \times m q} .
\end{aligned}
$$

Then we have the following result.

Proposition 18. The system modeled by (2) and (3) is null output controllable if and only if there exists $N \in \mathbb{N}_{+}$such that $C A^{N-1}=0$. In particular, if $A$ is nilpotent, then system (2) and (3) is null output controllable.

Proof.

Sufficiency. The general solution of (60) is given by

$$
\widetilde{x}_{i}=A^{i} \widetilde{x}_{0}+\sum_{j=0}^{i-1} A^{i-j-1} B u_{j}, \quad i \in \mathbb{N} .
$$


For $u_{i}=0, i \in \sigma_{0}^{N-1}$, we have $\tilde{x}_{N-1}=A^{N-1} \tilde{x}_{0}$, this implies that

$$
y_{N-1}=C \widetilde{x}_{N-1}+D_{0} u_{N-1}=C A^{N-1} \widetilde{x}_{0}+D_{0} u_{N-1}=0 ;
$$

since $C A^{N-1}=0$. Hence system (2) and (3) is null output controllable.

Necessity. System (2) and (3) is null output controllable, according to Proposition $17, \mathbb{Q}_{N}=0$ for some $N \in \mathbb{N}_{+}$. For $u_{0}^{N}=0$, we have

$$
y_{N-1}=\mathscr{Q}_{N} \widetilde{x}_{0}+\mathscr{R}_{N} u_{0}^{N}=0 \quad \forall \widetilde{x}_{0} \in \mathbb{R}_{+}^{n(p+1)+m q} .
$$

On the other hand, we have $y_{N-1}=C A^{N-1} \tilde{x}_{0}=0$; then $C A^{N-1}=0$ since $\widetilde{x}_{0}$ is arbitrary. This completes the proof.

In the remainder of this section and without loss of generality, we assume that $p \geq q$. Indeed, if $p<q$ we can set $A_{j}=0$ for $j \in \sigma_{p+1}^{q}$.

Lemma 19. For all $i \geq p$, we have

$$
A^{i}=\left(\begin{array}{ccccccc}
H_{i}^{0} & H_{i}^{1} & \cdots & H_{i}^{p} & L_{i}^{1} & \cdots & L_{i}^{q} \\
H_{i-1}^{0} & H_{i-1}^{1} & \cdots & H_{i-1}^{p} & L_{i-1}^{1} & \cdots & L_{i-1}^{q} \\
\vdots & \vdots & & \vdots & \vdots & & \vdots \\
H_{i-p}^{0} & H_{i-p}^{1} & \cdots & H_{i-p}^{p} & L_{i-p}^{1} & \cdots & L_{i-p}^{q} \\
0 & \cdots & \cdots & 0 & 0 & \cdots & 0 \\
\vdots & & & \vdots & \vdots & & \vdots \\
0 & \cdots & \cdots & 0 & 0 & \cdots & 0
\end{array}\right) .
$$

Proof. Let $u_{i}=0$ for $i \in \mathbb{N}$. Then, according to (64), we have

$$
\tilde{x}_{i}=A^{i} \tilde{x}_{0}, \quad i \in \mathbb{N} .
$$

On the other hand, from (18), for all $i \geq p$ we have

$$
\begin{aligned}
& \widetilde{x}_{i}=\left(\begin{array}{c}
x_{i} \\
x_{i-1} \\
\vdots \\
x_{i-p} \\
0 \\
\vdots \\
0
\end{array}\right) \\
& =\left(\begin{array}{ccccccc}
H_{i}^{0} & H_{i}^{1} & \cdots & H_{i}^{p} & L_{i}^{1} & \cdots & L_{i}^{q} \\
H_{i-1}^{0} & H_{i-1}^{1} & \cdots & H_{i-1}^{p} & L_{i-1}^{1} & \cdots & L_{i-1}^{q} \\
\vdots & \vdots & & \vdots & \vdots & & \vdots \\
H_{i-p}^{0} & H_{i-p}^{1} & \cdots & H_{i-p}^{p} & L_{i-p}^{1} & \cdots & L_{i-p}^{q} \\
0 & \cdots & \cdots & 0 & 0 & \cdots & 0 \\
\vdots & & & \vdots & \vdots & & \vdots \\
0 & \cdots & \cdots & 0 & 0 & \cdots & 0
\end{array}\right)\left(\begin{array}{c}
x_{0} \\
x_{-1} \\
\vdots \\
x_{-p} \\
u_{-1} \\
\vdots \\
u_{-q}
\end{array}\right) .
\end{aligned}
$$

Hence by identification between (68) and (69), we get that (67) holds.

Proposition 20. If, for some $s \in \sigma_{0}^{l}, C_{s}$ is injective, that is, rank $C_{s}=n$, then system (2) and (3) is null output controllable implying that $A$ is a nilpotent matrix.

Proof. System (2) and (3) is null output controllable; then by Proposition 17, for some $N \in \mathbb{N}_{+}$, we have $Q_{N}=0$. If $N \leq l$, then $\mathscr{H}_{N-1}^{j}=0, j \in \sigma_{0}^{p}, C_{k}=0, k \in \sigma_{N}^{l}$ and $\mathscr{L}_{N-1}^{k}=$ $0, k \in \sigma_{1}^{q}, D_{j}=0, j \in \sigma_{N}^{l}$. Then $s \in \sigma_{0}^{N-1}$ and $C_{s} H_{N-1-s}^{j}=$ $0, C_{s} L_{N-1-s}^{k}=0$. Since $C_{s}$ is injective, then $C_{s}^{T} C_{s}$ is invertible, which implies that $H_{N-1-s}^{j}=0$ and $L_{N-1-s}^{k}=0$. By Lemma 7, for $i \in \sigma_{0}^{p}$ we get $H_{N-1-s+i}^{j}=0$ and $L_{N-1-s+i}^{k}=0$. According to Lemma 19 , we have $A^{N-1-s+p}=0$, that is, $A$ is nilpotent. Similarly, we prove that $A$ is nilpotent if $N \geq 1+l$. This finishes the proof.

\section{Output Controllability}

By generalization of definition given in [11] we shall formulate the fundamental definitions for output controllability of system (2) and (3) as follows.

Definition 21. The system modeled by (2) and (3) is said to be output controllable in $N \in \mathbb{N}_{+}$steps if for any nonnegative initial state sequence $x_{-j} \in \mathbb{R}_{+}^{n}\left(j \in \sigma_{0}^{p}\right)$ and any nonnegative initial input sequence $u_{-j} \in \mathbb{R}_{+}^{m}\left(j \in \sigma_{1}^{q}\right)$, there exists a nonnegative input sequence $u_{i} \in \mathbb{R}_{+}^{m}, i \in \sigma_{0}^{N-1}$, which steers the output of the system from $x_{-j}$ to any desired nonnegative final output $y_{f} \in \mathbb{R}_{+}^{r}$, i.e., $y_{N-1}=y_{f}$.

Definition 22. The system modeled by (2) and (3) is said to be output controllable if there exists a positive integer $N \in \mathbb{N}_{+}$ such that the system is output controllable in $N$ steps.

The characterization of the output controllability is given by the following proposition.

Proposition 23. The system modeled by (2) and (3) is output controllable if and only if it is output reachable and null output controllable.

Proof.

Necessity. It is evident.

Sufficiency. Since system (2) and (3) is output reachable, then, according to Proposition 13, $\mathscr{R}_{N_{1}}$ for some $N_{1} \in \mathbb{N}_{+}$ includes a monomial submatrix of order $r \times r$. On the other hand, system (2) and (3) is null output controllable; hence, according to Proposition 17, $Q_{N_{2}}=0$ for some $N_{2} \in \mathbb{N}_{+}$. Then, for $N=\max \left\{N_{1}, N_{2}\right\}$, the matrix

$$
\mathscr{R}_{N}=\left(\begin{array}{ll}
\widetilde{R} & \mathscr{R}_{N_{1}}
\end{array}\right)
$$

contains a monomial submatrix of order $r \times r$, with $\widetilde{\mathscr{R}} \epsilon$ $\mathbb{R}_{+}^{r \times\left(N-N_{1}\right) m}$. Hence, by proof of Proposition 13 , for any $y_{f} \in$ 
$\mathbb{R}_{+}^{r}$, there exists a nonnegative input $u_{0}^{N} \in \mathbb{R}_{+}^{N m}$ such that

$$
y_{f}=\mathscr{R}_{N} u_{0}^{N} .
$$

And by Lemma 8, we have $\mathbb{Q}_{N}=0$. Then for every $\widetilde{x}_{0} \epsilon$ $\mathbb{R}_{+}^{n(p+1)+m q}$ we get that

$$
y_{N-1}=\mathscr{Q}_{N} \tilde{x}_{0}+\mathscr{R}_{N} u_{0}^{N}=y_{f} ;
$$

that is, system (2) and (3) is output controllable. The proposition is proved.

\section{Numerical Examples}

Example 1 (output reachability). Suppose that we are given system (2) and (3) with $p=q=l=2$ and matrices

$$
\begin{aligned}
& A_{0}=\left(\begin{array}{lll}
0 & 0 & 0 \\
0 & 0 & 0 \\
0 & 0 & 1
\end{array}\right), \\
& A_{1}=\left(\begin{array}{lll}
0 & 0 & 0 \\
0 & 1 & 0 \\
0 & 0 & 0
\end{array}\right) \text {, } \\
& A_{2}=\left(\begin{array}{lll}
0 & 0 & 0 \\
0 & 1 & 0 \\
1 & 1 & 0
\end{array}\right), \\
& B_{0}=\left(\begin{array}{l}
0 \\
1 \\
0
\end{array}\right) \text {, } \\
& B_{1}=\left(\begin{array}{l}
1 \\
0 \\
0
\end{array}\right) \text {, } \\
& B_{2}=\left(\begin{array}{l}
0 \\
1 \\
0
\end{array}\right) \text {, } \\
& C_{0}=\left(\begin{array}{lll}
1 & 0 & 0 \\
0 & 0 & 1
\end{array}\right), \\
& C_{1}=\left(\begin{array}{lll}
0 & 0 & 1 \\
0 & 0 & 0
\end{array}\right), \\
& C_{2}=\left(\begin{array}{lll}
0 & 0 & 0 \\
0 & 0 & 1
\end{array}\right), \\
& D_{0}=\left(\begin{array}{l}
1 \\
0
\end{array}\right) \text {, }
\end{aligned}
$$

$$
\begin{aligned}
D_{1} & =\left(\begin{array}{l}
0 \\
0
\end{array}\right), \\
D_{2} & =\left(\begin{array}{l}
1 \\
0
\end{array}\right) .
\end{aligned}
$$

The conditions of Proposition 13 are satisfied because the output reachability matrix in five steps

$$
\mathscr{R}_{5}=\left(\begin{array}{lllll}
\mathscr{K}_{3} & \mathscr{K}_{2} & \overline{\mathscr{K}}_{1} & \overline{\mathscr{K}}_{0} & D_{0}
\end{array}\right)=\left(\begin{array}{lllll}
0 & 0 & 2 & 0 & 1 \\
1 & 0 & 0 & 0 & 0
\end{array}\right)
$$

contains a monomial submatrix of order $2 \times 2$.

By simple calculation, we get

$$
\mathscr{R}_{5}^{T}\left(\mathscr{R}_{5} \mathscr{R}_{5}^{T}\right)^{-1}=\left(\begin{array}{cc}
0 & 1 \\
0 & 0 \\
0.4 & 0 \\
0 & 0 \\
0.2 & 0
\end{array}\right) \in \mathbb{R}_{+}^{5 \times 2}
$$

Then the nonnegative input sequence that permitted to transfer the output from the zero initial conditions to the final output $y_{f}=\left(\begin{array}{ll}1 & 0.5\end{array}\right)^{T}$ according to (56) is

$$
u_{0}^{5}=\mathscr{R}_{5}^{T}\left(\mathscr{R}_{5} \mathscr{R}_{5}^{T}\right)^{-1} y_{f}=\left(\begin{array}{lllll}
0.5 & 0 & 0.4 & 0 & 0.2
\end{array}\right)^{T} .
$$

Table 1 gives the values of the output at each step. We see that the final output has been reached within a number of steps of the input data sequence greater than $n+1=4$.

This comes up to be a particularity of discrete delay systems. This is not satisfied in the case of discrete systems without delay where the steps to reach the final output $y_{f}$ are always less than or equal to $n+1$. This results from the CayleyHamilton theorem.

The next two examples study, respectively, the conditions of the null output controllability and output controllability.

Example 2 (null output controllability). Consider the system modeled by (2) and (3) with matrices

$$
\begin{aligned}
& A_{0}=\left(\begin{array}{lll}
0 & 0 & 0 \\
0 & 0 & 0 \\
1 & 0 & 0
\end{array}\right), \\
& A_{1}=\left(\begin{array}{lll}
0 & 0 & 0 \\
0 & 0 & 0 \\
1 & 0 & 0
\end{array}\right), \\
& A_{2}=\left(\begin{array}{lll}
0 & 0 & 0 \\
2 & 0 & 0 \\
0 & 0 & 0
\end{array}\right),
\end{aligned}
$$


TABLE 1: Values of the outputs in the transfer steps.

\begin{tabular}{lcccc}
\hline$N$ & 1 & 2 & 3 & 4 \\
\hline$y_{N-1}$ & $\left(\begin{array}{c}0.5 \\
0\end{array}\right)$ & $\left(\begin{array}{c}0.5 \\
0\end{array}\right)$ & $\left(\begin{array}{c}1.4 \\
0\end{array}\right)$ & $\left(\begin{array}{l}0 \\
0\end{array}\right)$ \\
\hline
\end{tabular}

$$
\begin{aligned}
& B_{1}=\left(\begin{array}{l}
0 \\
1 \\
0
\end{array}\right), \\
& B_{2}=\left(\begin{array}{l}
0 \\
0 \\
2
\end{array}\right), \\
& C_{0}=\left(\begin{array}{lll}
3 & 0 & 0 \\
0 & 0 & 1
\end{array}\right), \\
& C_{1}=\left(\begin{array}{lll}
1 & 0 & 0 \\
1 & 0 & 0
\end{array}\right), \\
& C_{2}=\left(\begin{array}{lll}
2 & 0 & 0 \\
0 & 0 & 0
\end{array}\right) .
\end{aligned}
$$

System (2) and (3) is null output controllable because the null output controllability matrix in four steps

$$
Q_{4}=\left(\begin{array}{lllll}
\mathscr{H}_{3}^{0} & \mathscr{H}_{3}^{1} & \mathscr{H}_{3}^{2} & \mathscr{L}_{3}^{1} & \mathscr{L}_{3}^{2}
\end{array}\right)
$$

is null.

System (2), (3) in this example is null output controllable for any $C_{k} \in \mathbb{R}_{+}^{r \times 3}\left(k \in \sigma_{0}^{2}\right)$ because the matrix $A$ is nilpotent with index $k=6$; that is, $A^{k-1} \neq 0$ and $A^{k}=0$.

Example 3 (output controllability). Consider the system modeled by (2) and (3) with matrices

$$
\begin{aligned}
& A_{0}=\left(\begin{array}{lll}
0 & 0 & 0 \\
1 & 0 & 0 \\
1 & 0 & 0
\end{array}\right), \\
& A_{1}=\left(\begin{array}{lll}
0 & 0 & 0 \\
0 & 0 & 0 \\
1 & 1 & 0
\end{array}\right), \\
& A_{2}=\left(\begin{array}{lll}
0 & 0 & 0 \\
0 & 0 & 0 \\
1 & 0 & 1
\end{array}\right), \\
& B_{0}=\left(\begin{array}{ll}
0 & 0 \\
1 & 0 \\
0 & 0
\end{array}\right),
\end{aligned}
$$

$$
\begin{aligned}
& B_{1}=\left(\begin{array}{ll}
0 & 0 \\
1 & 2 \\
0 & 0
\end{array}\right), \\
& B_{2}=\left(\begin{array}{ll}
0 & 0 \\
0 & 0 \\
1 & 3
\end{array}\right), \\
& C_{0}=\left(\begin{array}{lll}
0 & 0 & 0 \\
1 & 0 & 0
\end{array}\right), \\
& C_{1}=\left(\begin{array}{lll}
1 & 0 & 0 \\
0 & 0 & 0
\end{array}\right), \\
& C_{2}=\left(\begin{array}{lll}
1 & 0 & 0 \\
1 & 0 & 0
\end{array}\right), \\
& D_{0}=\left(\begin{array}{ll}
1 & 4 \\
0 & 3
\end{array}\right), \\
& D_{2}=\left(\begin{array}{ll}
0 & 2 \\
1 & 1
\end{array}\right) . \\
& D_{1}=\left(\begin{array}{ll}
1 & 1 \\
0 & 1
\end{array}\right),
\end{aligned}
$$

System (2) and (3) is output reachable because the output reachability matrix in tree steps

$$
\mathscr{R}_{3}=\left(\begin{array}{lll}
\overline{\mathscr{K}}_{1} & \overline{\mathscr{K}}_{0} & D_{0}
\end{array}\right)=\left(\begin{array}{llllll}
0 & 2 & 1 & 1 & 1 & 4 \\
1 & 1 & 0 & 1 & 0 & 3
\end{array}\right)
$$

contains a monomial submatrix of order $2 \times 2$.

The conditions of Proposition 17 are satisfied because the null output controllability matrix in four steps

$$
Q_{4}=\left(\begin{array}{lllll}
\mathscr{H}_{3}^{0} & \mathscr{H}_{3}^{1} & \mathscr{H}_{3}^{2} & \mathscr{L}_{3}^{1} & \mathscr{L}_{3}^{2}
\end{array}\right)
$$

is null, so by proof of Proposition 23, the system is output controllable in four steps.

\section{Conclusion}

The output controllability of positive discrete linear systems with delays in state, control, and output has been considered. Necessary and sufficient conditions for the positivity of discrete systems have been established (Proposition 4). Criteria for output reachability (Proposition 13) and null 
output controllability (Proposition 17) of the positive discrete systems have been also proved. It has been shown that output reachability and null output controllability together imply output controllability (Proposition 23). Numerical examples were given to illustrate the results.

We think that the techniques used in this paper can be useful to investigate the output reachability, null output controllability, and output controllability problems for different positive dynamical systems such as switched systems, fractional systems with different orders, and fractional switched systems.

\section{Conflicts of Interest}

The authors declare that there are no conflicts of interest regarding the publication of this paper.

\section{References}

[1] R. E. Kalman, "On the general theory of control systems," in Proceeding of the First International Congress on Automatic Control, vol. 1, pp. 481-493, Butterworth, London, UK, 1960.

[2] C. T. Chen, Introduction to Linear System Theory, Holt, Rinehart and Winston, NY, USA, 1970.

[3] J. Klamka, Controllability of dynamical systems, vol. 48, Kluwer Academic Publishers, Dordrecht, The Netherlands, 1991.

[4] J. Klamka, "Controllability of dynamical systems. A survey," Bulletin of the Polish Academy of Sciences: Technical Sciences, vol. 61, no. 2, pp. 335-342, 2013.

[5] A. Bacciotti and L. Mazzi, "Asymptotic controllability by means of eventually periodic switching rules," SIAM Journal on Control and Optimization, vol. 49, no. 2, pp. 476-497, 2011.

[6] K. Balachandran, J. Kokila, and J. J. Trujillo, "Relative controllability of fractional dynamical systems with multiple delays in control," Computers \& Mathematics with Applications, vol. 64, no. 10, pp. 3037-3045, 2012.

[7] J. Klamka, "Constrained controllability of second order dynamical systems with delay," Control and Cybernetics, vol. 42, no. 1, pp. 111-121, 2013.

[8] L. Shen, J. Shi, and J. Sun, "Complete controllability of impulsive stochastic integro-differential systems," Automatica, vol. 46, no. 6, pp. 1068-1073, 2010.

[9] L. Shen and J. Sun, "Approximate controllability of stochastic impulsive functional systems with infinite delay," Automatica, vol. 48, no. 10, pp. 2705-2709, 2012.

[10] L. Tie, "On small-controllability and controllability of a class of nonlinear systems," International Journal of Control, vol. 87, no. 10, pp. 2167-2175, 2014.

[11] A. Babiarz, A. Czornik, and M. Niezabitowski, "Output controllability of the discrete-time linear switched systems," Nonlinear Analysis: Hybrid Systems, vol. 21, pp. 1-10, 2016.

[12] M. I. Garca-Planas and J. L. Domnguez-Garca, "Alternative tests for functional and pointwise output-controllability of linear time-invariant systems," Systems \& Control Letters, vol. 62, no. 5, pp. 382-387, 2013.

[13] D. Lau, D. Oetomo, and S. K. Halgamuge, "Generalized modeling of multilink cable-driven manipulators with arbitrary routing using the cable-routing matrix," IEEE Transactions on Robotics, vol. 29, no. 5, pp. 1102-1113, 2013.
[14] J. L. Domnguez-Garca and M. I. Garca-Planas, "Functional output-controllability analysis of fixed speed wind turbine," in Proceedings of the 6th International Conference on Physics and Control, San Luis Potosí, Mexico, 2013.

[15] M. I. Garcia-Planas and S. Tarragona, "Analysis of functional output controllability of time-invariant composite linear systems," Recent Advances in Systems, Control and Informatics, pp. 40-47, 2013.

[16] D. G. Luenberger, Introduction to Dynamic Systems: Theory, Models and Applications, Academic Press, New York, NY, USA, 1979.

[17] L. Farina and S. Rinaldi, Positive Linear Systems: Theory and Applications, John Wiley, New York, NY, USA, 2000.

[18] T. Kaczorek, "Positive 1D and 2D systems," in Communications and Control Engineering, Springer-Verlag, London, UK, 2002.

[19] C. Briat, "Dwell-time stability and stabilization conditions for linear positive impulsive and switched systems," Nonlinear Analysis: Hybrid Systems, vol. 24, pp. 198-226, 2017.

[20] J. Shen and J. Lam, "On $l_{\infty}$ and $L_{\infty}$ gains for positive systems with bounded time-varying delays," International Journal of Systems Science, vol. 46, pp. 1-8, 2015.

[21] P. G. Coxson and H. Shapiro, "Positive input reachability and controllability of positive systems," Linear Algebra and its Applications, vol. 94, pp. 35-53, 1987.

[22] M. P. Fanti, B. Maione, and B. Turchiano, "Controllability of multi-input positive discrete-time systems," International Journal of Control, vol. 51, no. 6, pp. 1295-1308, 1990.

[23] D. N. P. Murthy, "Controllability of a linear positive dynamic system," International Journal of Systems Science, vol. 17, no. 1, pp. 49-54, 1986.

[24] V. G. Rumchev and D. J. James, "Controllability of positive linear discrete-time systems," International Journal of Control, vol. 50, no. 3, pp. 845-857, 1989.

[25] L. Caccetta and V. G. Rumchev, "A survey of reachability and controllability for positive linear systems," Annals of Operations Research, vol. 98, pp. 101-122, 2000.

[26] M. E. Valcher, "Controllability and reachability criteria for discrete time positive systems," International Journal of Control, vol. 65, no. 3, pp. 511-536, 1996.

[27] G. Xie and L. Wang, "Reachability and controllability of positive linear discrete-time systems with time-delays," in Positive systems (Rome, 2003), L. Benvenuti, A. De Santis, and L. Farina, Eds., vol. 294, pp. 377-384, Springer, Berlin, Germany, 2003.

[28] T. Kaczorek, "Output-reachability of positive linear discretetime systems," in Proceeding of 7th International Workshop, Computational Problems of Electrical Engineering, (CPEE '06), pp. 64-68, Odessa, Ukraine, 2006.

[29] T. Kaczorek, "Output-reachability of positive linear discretetime systems with delays," Archives of Control Sciences, vol. 16, no. 3, pp. 247-255, 2006.

[30] A. Berman and R. J. Plemmons, Nonnegative Matrices in the Mathematical Sciences, vol. 9, Society for Industrial and Applied Mathematics, Philadelphia, Pa, USA, 1994.

[31] M. Buslowicz, "On some properties of the solution of state equation of discrete-time systems with delays," Zesz. Nauk. Polit. Bial., Elektrotechnika, vol. 1, pp. 17-29, 1983 (Polish). 


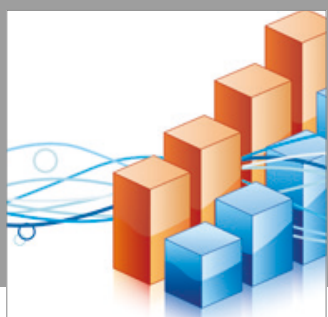

Advances in

Operations Research

vatersals

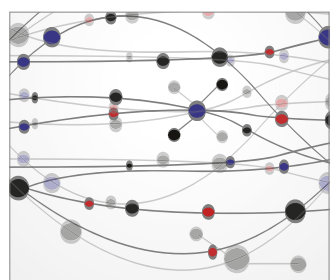

\section{The Scientific} World Journal
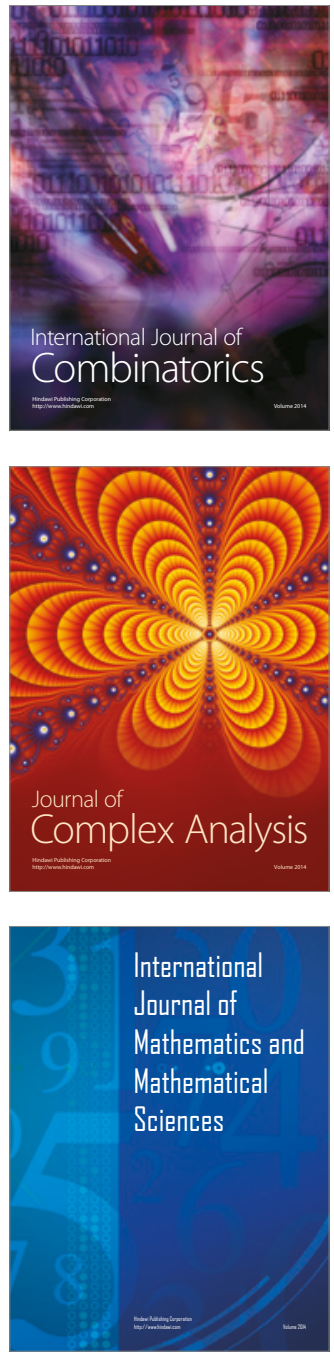
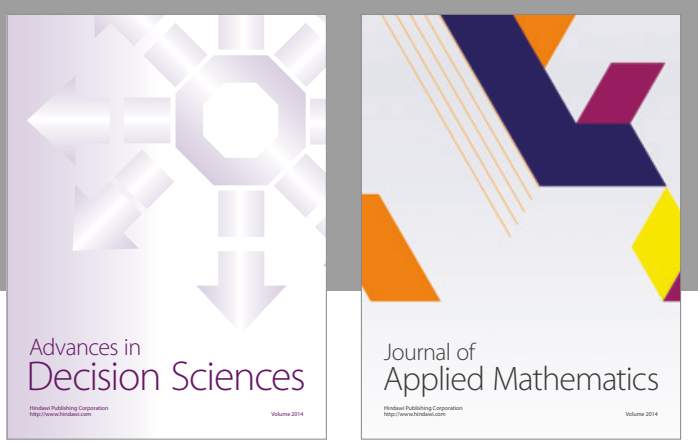

Algebra

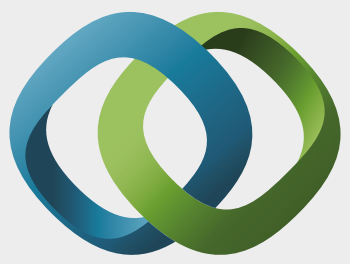

\section{Hindawi}

Submit your manuscripts at

https://www.hindawi.com
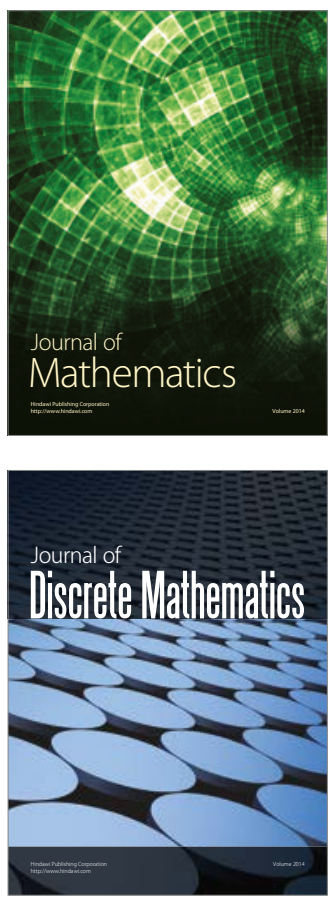

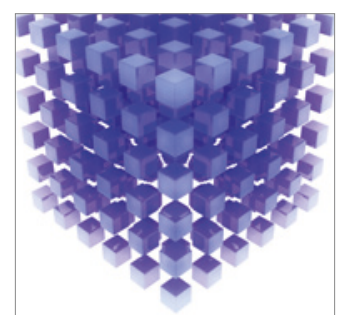

Mathematical Problems in Engineering
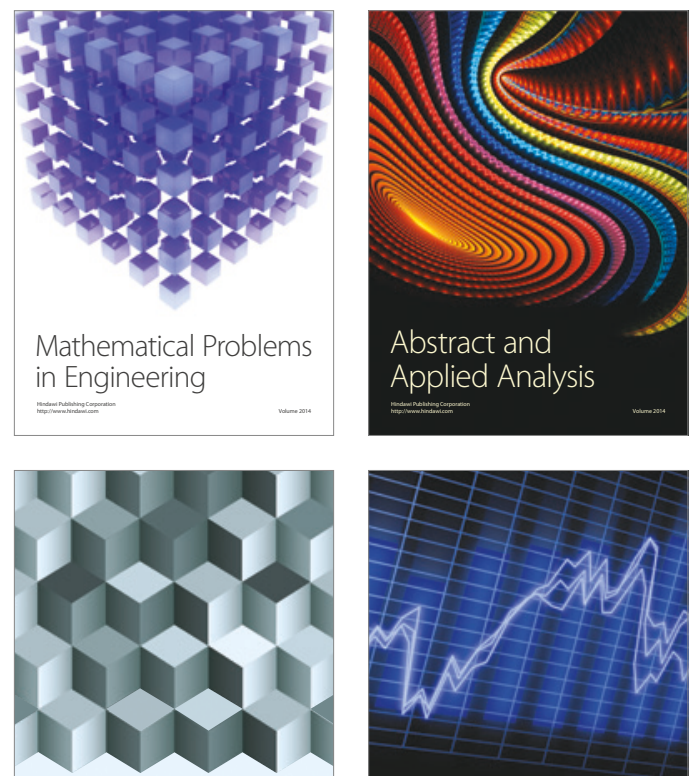

Journal of

Function Spaces

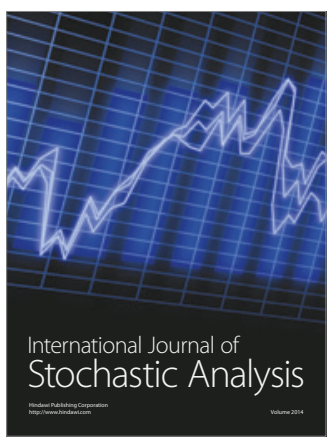

Probability and Statistics
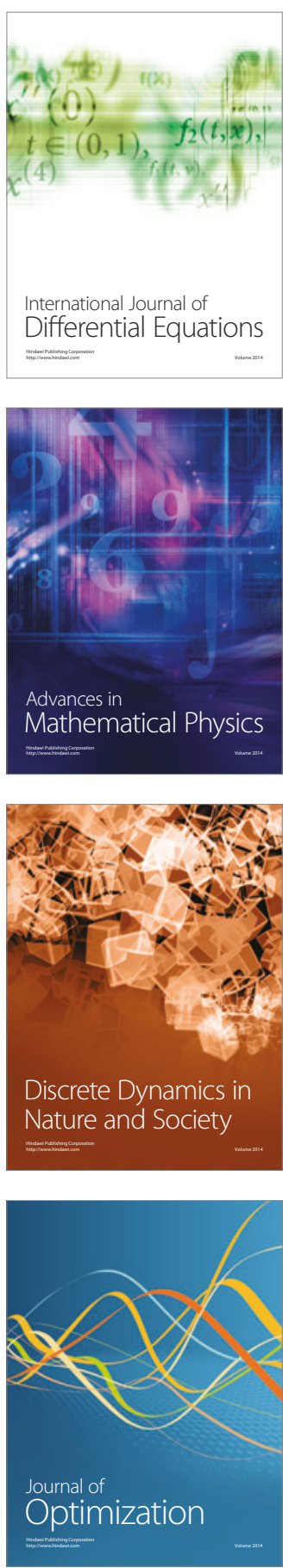